\title{
A normalization scheme for the non-symmetric s-step Lanczos algorithm
}

\section{Conference Paper}

\section{Author(s):}

Feuerriegel, Stefan; Bücker, H.M.

Publication date:

2013

\section{Permanent link:}

https://doi.org/10.3929/ethz-b-000182415

Rights / license:

In Copyright - Non-Commercial Use Permitted

\section{Originally published in:}

Lecture Notes in Computer Science 8286, https://doi.org/10.1007/978-3-319-03889-6_4 


\title{
A Normalization Scheme for the Non-Symmetric $s$-Step Lanczos Algorithm
}

\author{
Stefan Feuerriegel ${ }^{1}$ and H. Martin Bücker ${ }^{2}$ \\ 1 University of Freiburg, 79098 Freiburg, Germany \\ 2 Friedrich Schiller University Jena, 07743 Jena, Germany
}

\begin{abstract}
The Lanczos algorithm is among the most frequently used techniques for computing a few dominant eigenvalues of a large sparse non-symmetric matrix. When variants of this algorithm are implemented on distributed-memory computers, the synchronization time spent in computing dot products is increasingly limiting the parallel scalability. The goal of $s$-step algorithms is to reduce the harmful influence of dot products on the parallel performance by grouping several of these operations for joint execution; thus, plummeting synchronization time when using a large number of processes. This paper extends the non-symmetric $s$-step Lanczos method introduced by Kim and Chronopoulos (J. Comput. Appl. Math., 42(3), 357-374, 1992) by a novel normalization scheme. Compared to the unnormalized algorithm, the normalized variant improves numerical stability and reduces the possibility of breakdowns.
\end{abstract}

Keywords: $s$-step Lanczos, numerical stability, synchronization-reducing

\section{Introduction}

Non-symmetric eigenvalue problems arising from computational science and engineering are often large and sparse. When only a few dominant eigenvalues are needed, iterative Krylov methods enter the picture, e. g., the Lanczos algorithm [1]. When parallelizing the Lanczos method on message-passing architectures, naïve approaches focus on balancing computational load among processes, but mainly ignore communication and synchronization. These approaches essentially consist of parallelizing a known serial iterative method by parallelizing each linear algebra operation individually. Thus, the resulting implementations inherit most of the properties of the given algorithms including their serial nature. Since this often leads to poor performance, significant research effort is spent in designing new Krylov algorithms specifically for parallel computers: (i) Communication-overlapping algorithms $[2,3]$ aim at reducing the impact of a communication event by overlapping it with computation and/or other communication. (ii) Communication-avoiding algorithms $[4,5]$ rely on blocking to reduce the volume of communication. (iii) Synchronization-free algorithms [6] do not involve any synchronization of all processes at the same time. (iv) Synchronizationreducing algorithms [7-11] try to minimize the number of global synchronization 
points (GSP), locations of an algorithm at which all information local to a process has to be globally available on all processes in order to continue the computation.

While communication-avoiding algorithms successfully reduce communication volume between processes, they do not directly focus on synchronization among processes. However, synchronization will increasingly dominate the total execution time on future extreme-scale computer systems where the number of processes will be huge. Therefore, we focus on a novel synchronization-reducing Krylov algorithm. Here, a GSP is enforced by dot product-like operations involving a reduction operation on all participating processes. When only a single GSP is enforced for $s$ iterations of the corresponding classical algorithm, this synchronization-reducing algorithm is referred to as an $s$-step method [12-14]. The $s$-step Lanczos procedure was originally introduced for symmetric matrices [15] and later extended to non-symmetric matrices [16]. The contribution of the present paper is to derive a new variant of the algorithm [16] by extending it with a normalization scheme with an improved numerical stability. This different normalization scheme changes the underlying recurrences so that, in Sect. 2, a novel derivation of the complete algorithm is necessary. Sections 3 and 4 describe a numerical experiment and the resulting parallel performance.

Given two vectors, their dot product is denoted by $\langle\boldsymbol{v}, \boldsymbol{w}\rangle$. The symbol $0_{n, m}$ is used for an $n \times m$ zero matrix. Concatenation of scalar entries that form a row vector is denoted by $\left[x_{1}, \ldots, x_{n}\right]$. Concatenation of vectors or matrices that form a (block) matrix is indicated by $\left[\boldsymbol{v}_{1}\|\ldots\| \boldsymbol{v}_{n}\right]$.

\section{The $s$-Step Lanczos Method with Normalization}

In the classical Lanczos algorithm [1], every iteration computes a pair of individual Lanczos vectors $\boldsymbol{v}_{k}$ and $\boldsymbol{w}_{k}$ using a GSP. In contrast, the $k$-th block iteration of the $s$-step Lanczos algorithm [16] computes a pair of blocks of $s$ Lanczos vectors denoted by $\bar{V}_{k}=\left[\boldsymbol{v}_{k}^{1}\|\ldots\| \boldsymbol{v}_{k}^{s}\right] \in \mathbb{R}^{N \times s}$ and $\bar{W}_{k}=\left[\boldsymbol{w}_{k}^{1}\|\ldots\| \boldsymbol{w}_{k}^{s}\right] \in$ $\mathbb{R}^{N \times s}$. This way, a single block iteration of the $s$-step Lanczos algorithm generates $s$ iterations of the classical Lanczos algorithm using only a single GSP.

The $s$-step Lanczos method proceeds in two steps. First, relaxed Lanczos vectors are computed in a block-wise fashion. In each block iteration, a new block containing $s$ of these vectors as columns is computed. Second, a back transformation is then applied to these vectors.

Definition 1 ( $s$-Step Lanczos Algorithm). Let $n=s k$ with $1 \leq n \leq N$. For a given non-symmetric matrix $A \in \mathbb{R}^{N \times N}$, the s-step Lanczos algorithm generates an upper Hessenberg matrix $\ddot{T}_{n} \in \mathbb{R}^{n \times n}$ as well as two additional matrices $\ddot{V}_{n} \in \mathbb{R}^{N \times n}$ and $\ddot{W}_{n} \in \mathbb{R}^{N \times n}$ such that

$$
\begin{aligned}
\ddot{W}_{n}^{T} \ddot{V}_{n} & =\text { block biorthogonal } \\
A \ddot{V}_{n} & =\ddot{V}_{n} \ddot{T}_{n}+f_{k+1} \boldsymbol{v}_{k+1}^{1}[0, \ldots, 0,1], \\
A^{T} \ddot{W}_{n} & =\ddot{W}_{n} \ddot{T}_{n}+f_{k+1} \boldsymbol{w}_{k+1}^{1}[0, \ldots, 0,1] .
\end{aligned}
$$


The upper Hessenberg matrix $\ddot{T}_{n}$ is block tridiagonal

$$
\ddot{T}_{n}=\left[\begin{array}{ccc}
\bar{G}_{1} & \bar{E}_{2} & \\
\bar{F}_{2} \bar{G}_{2} & \ddots \\
\ddots & \ddots \\
& \bar{F}_{k} \bar{E}_{k}
\end{array}\right] \in \mathbb{R}^{n \times n} \text { where each } \bar{F}_{i}=\left[\quad f_{i}\right] \in \mathbb{R}^{s \times s},
$$

each $\bar{E}_{i}$ is a dense $s \times s$ matrix, and each $\bar{G}_{i} \in \mathbb{R}^{s \times s}$ is in upper Hessenberg form. The block-wise grouping of the relaxed Lanczos vectors is given by $\ddot{V}_{n}=\left[\bar{V}_{1}\|\ldots\| \bar{V}_{k}\right] \in \mathbb{R}^{N \times n}$ and $\ddot{W}_{n}=\left[\bar{W}_{1}\|\ldots\| \bar{W}_{k}\right] \in \mathbb{R}^{N \times n}$ where, for all $k$, we have $\bar{V}_{k}=\left[\boldsymbol{v}_{k}^{1}\|\ldots\| \boldsymbol{v}_{k}^{s}\right] \in \mathbb{R}^{N \times s}, \bar{W}_{k}=\left[\boldsymbol{w}_{k}^{1}\|\ldots\| \boldsymbol{w}_{k}^{s}\right] \in \mathbb{R}^{N \times s}$, $\boldsymbol{v}_{k}^{1}, \ldots, \boldsymbol{v}_{k}^{s} \in \mathbb{R}^{N}$ and $\boldsymbol{w}_{k}^{1}, \ldots, \boldsymbol{w}_{k}^{s} \in \mathbb{R}^{N}$.

In an implementation, the $s$-step Lanczos algorithm iterates up to a block iteration $k=n / s$ yielding both $\ddot{T}_{n}$ and $\ddot{V}_{n}$. It then turns $\ddot{T}_{n}$ and $\ddot{V}_{n}$ into the corresponding matrices computed by the classical Lanczos procedure. This back transformation is sketched in the following theorem whose proof is given in [16].

Theorem 1. Let $\ddot{W}_{n}^{T} \ddot{V}_{n}$ be a non-singular matrix and let $\ddot{W}_{n}^{T} \ddot{V}_{n}=\ddot{L}_{n} \ddot{U}_{n}$ denote its $L U$ decomposition. Then, $\ddot{T}_{n}, \ddot{V}_{n}$ and $\ddot{W}_{n}$ can be transformed into $T_{n}, W_{n}$ and $V_{n}$, originating from the classical Lanczos method in the absence of breakdowns, by $T_{n}:=\ddot{U}_{n} \ddot{T}_{n} \ddot{U}_{n}^{-1}, V_{n}:=\ddot{V}_{n} \ddot{U}_{n}^{-1}$ and $W_{n}^{T}:=\ddot{L}_{n}^{-1} \ddot{W}_{n}^{T}$.

To state computational schemes for $\bar{V}_{k+1}$ and $\bar{W}_{k+1}$, we introduce the column notation $\bar{E}_{k}=\left[\boldsymbol{e}_{k}^{1}\|\ldots\| \boldsymbol{e}_{k}^{s}\right]$ and $\bar{G}_{k}=\left[\boldsymbol{g}_{k}^{1}\|\ldots\| \boldsymbol{g}_{k}^{s}\right]$. Then, according to [17], the $s$-step basis vectors are computed as

$$
\begin{aligned}
\tilde{\boldsymbol{v}}_{k+1}^{1} & :=f_{k+1} \boldsymbol{v}_{k+1}^{1}=A \boldsymbol{v}_{k}^{s}-\bar{V}_{k-1} \boldsymbol{e}_{k}^{s}-\bar{V}_{k} \boldsymbol{g}_{k}^{s}, \\
\tilde{\boldsymbol{w}}_{k+1}^{1} & :=f_{k+1} \boldsymbol{w}_{k+1}^{1}=A^{T} \boldsymbol{w}_{k}^{s}-\bar{W}_{k-1} \boldsymbol{e}_{k}^{s}-\bar{W}_{k} \boldsymbol{g}_{k}^{s} .
\end{aligned}
$$

Here, in contrast to [16], we can choose the coefficient $f_{k+1}$ arbitrarily to transform the vectors $\tilde{\boldsymbol{v}}_{k+1}^{1}$ and $\tilde{\boldsymbol{w}}_{k+1}^{1}$ into normalized vectors $\boldsymbol{v}_{k+1}^{1}$ and $\boldsymbol{w}_{k+1}^{1}$.

To compute the remaining Lanczos vectors of the current block, [17] uses plain orthogonalization against $\bar{V}_{k}$ or $\bar{W}_{k}$ arriving at

$$
\begin{array}{ll}
\tilde{\boldsymbol{v}}_{k+1}^{j}=A^{j-1} \boldsymbol{v}_{k+1}^{1}-\bar{V}_{k} \boldsymbol{t}_{k}^{j}, & \text { for } j=2, \ldots, s, \\
\tilde{\boldsymbol{w}}_{k+1}^{j}=\left(A^{T}\right)^{j-1} \boldsymbol{w}_{k+1}^{1}-\bar{W}_{k} \hat{\boldsymbol{t}}_{k}^{j}, & \text { for } j=2, \ldots, s .
\end{array}
$$

Here, the vectors $\boldsymbol{t}_{k}^{j}$ and $\hat{\boldsymbol{t}}_{k}^{j}$ are determined to match the conditions $\bar{V}_{k+1} \perp \bar{W}_{k}$ and $\bar{W}_{k+1} \perp \bar{V}_{k}$ which is sufficient to enforce the block biorthogonality (1a) as stated in the following theorem whose proof is given in [16].

Theorem 2. If $\boldsymbol{v}_{1}^{1}=\boldsymbol{w}_{1}^{1}$ holds, then $\boldsymbol{t}_{k}^{j}=\hat{\boldsymbol{t}}_{k}^{j}$ for $j=2, \ldots, s$ and the matrices $\ddot{V}_{n+s}$ and $\ddot{W}_{n+s}$ with $n=$ sk are block biorthogonal. 
When we implemented the original $s$-step Lanczos algorithm [16] in double precision floating-point arithmetic, we experienced that numerical overflows can occur. We observed that the floating-point values in $\ddot{W}_{n}^{T} \ddot{V}_{n}$ grew rapidly, quickly leaping the maximum value in floating-point arithmetic. To reduce the possibility of numerical overflows, we introduce the normalization scheme

$$
f_{k+1}:=\sqrt{\left|\left\langle\tilde{\boldsymbol{w}}_{k+1}^{1}, \tilde{\boldsymbol{v}}_{k+1}^{1}\right\rangle\right|}
$$

such that $\left\langle\boldsymbol{w}_{k+1}^{1}, \boldsymbol{v}_{k+1}^{1}\right\rangle= \pm 1$. This differs from [16] where any normalization is avoided, corresponding to $f_{k+1}:=1$ in the new scheme.

In addition to this normalization scheme, it turns out that it is also convenient to normalize $\tilde{\boldsymbol{v}}_{k+1}^{j}$ and $\tilde{\boldsymbol{w}}_{k+1}^{j}$ for $j=2, \ldots, s$ such that $\left\langle\boldsymbol{w}_{k+1}^{j}, \boldsymbol{v}_{k+1}^{j}\right\rangle= \pm 1$ holds. Therefore, we scale the Lanczos vectors as follows:

$$
\boldsymbol{v}_{k+1}^{j}=\tilde{\boldsymbol{v}}_{k+1}^{j} \sigma_{k+1}^{j} \quad \text { and } \quad \boldsymbol{w}_{k+1}^{j}=\tilde{\boldsymbol{w}}_{k+1}^{j} \sigma_{k+1}^{j} \quad \text { for } \quad j=2, \ldots, s,
$$

where

$$
\sigma_{k+1}^{j}:=1 / \sqrt{\left|\left\langle\tilde{\boldsymbol{w}}_{k+1}^{j}, \tilde{\boldsymbol{v}}_{k+1}^{j}\right\rangle\right|} \text { for } j=2, \ldots, s .
$$

To compute the vectors $\boldsymbol{e}_{k}^{i}, \boldsymbol{g}_{k}^{i}$ and $\boldsymbol{t}_{k}^{j}$, we let the matrix $\bar{M}_{k}:=\bar{W}_{k}^{T} \bar{V}_{k}$ be non-singular. Then, according to [17], we find these vectors as the solutions of the following $s \times s$ systems of linear equations:

$$
\begin{aligned}
\bar{M}_{k-1} \boldsymbol{e}_{k}^{i} & =\boldsymbol{c}_{k}^{i} \text { where } \boldsymbol{c}_{k}^{i}=\left[\left\langle\boldsymbol{w}_{k-1}^{1}, A \boldsymbol{v}_{k}^{i}\right\rangle, \ldots,\left\langle\boldsymbol{w}_{k-1}^{s}, A \boldsymbol{v}_{k}^{i}\right\rangle\right]^{T}, \\
\bar{M}_{k} \boldsymbol{g}_{k}^{i} & =\boldsymbol{d}_{k}^{i} \text { where } \boldsymbol{d}_{k}^{i}=\left[\left\langle\boldsymbol{w}_{k}^{1}, A \boldsymbol{v}_{k}^{i}\right\rangle, \ldots,\left\langle\boldsymbol{w}_{k}^{s}, A \boldsymbol{v}_{k}^{i}\right\rangle\right]^{T}, \\
\bar{M}_{k} \boldsymbol{t}_{k}^{j} & =\boldsymbol{b}_{k}^{j} \text { where } \boldsymbol{b}_{k}^{j}=\left[\left\langle\boldsymbol{w}_{k}^{1}, A^{j-1} \boldsymbol{v}_{k+1}^{1}\right\rangle, \ldots,\left\langle\boldsymbol{w}_{k}^{s}, A^{j-1} \boldsymbol{v}_{k+1}^{1}\right\rangle\right]^{T},
\end{aligned}
$$

where $i=1, \ldots, s$ and $j=2, \ldots, s$. Computing the dot products $\boldsymbol{b}_{k}^{i, j}:=$ $\left\langle\boldsymbol{w}_{k}^{i}, A^{j-1} \boldsymbol{v}_{k+1}^{1}\right\rangle, \boldsymbol{c}_{k}^{i, j}:=\left\langle\boldsymbol{w}_{k-1}^{i}, A \boldsymbol{v}_{k}^{j}\right\rangle, \boldsymbol{d}_{k}^{i, j}:=\left\langle\boldsymbol{w}_{k}^{i}, A \boldsymbol{v}_{k}^{j}\right\rangle$ and $\bar{M}_{k}^{i, j}:=\left\langle\boldsymbol{w}_{k}^{i}, \boldsymbol{v}_{k}^{j}\right\rangle$ explicitly for all $i, j=1, \ldots, s$ is a computationally expensive task that would destroy all benefits from the $s$-step approach at once. Fortunately, as detailed in [17], there is a remedy to this problem consisting of computing these products recursively from the $2 s$ dot products $\left\langle\boldsymbol{w}_{k}^{1}, \boldsymbol{v}_{k}^{1}\right\rangle,\left\langle\boldsymbol{w}_{k}^{1}, A \boldsymbol{v}_{k}^{1}\right\rangle, \ldots,\left\langle\boldsymbol{w}_{k}^{1}, A^{2 s-1} \boldsymbol{v}_{k}^{1}\right\rangle$.

Putting everything together, we now finalize the $s$-step Lanczos algorithm with normalization. Recall from Theorem 2 that the iteration is started with two identical vectors. We emphasize in Step 11 of the following algorithm that, for each pair of $s$ Lanczos vectors, only a single GSP is required.

Input: Matrix $A \in \mathbb{R}^{N \times N}$, starting vectors $\boldsymbol{v}_{1}^{1}=\boldsymbol{w}_{1}^{1}$.

Output: Tridiagonal matrix $T_{n} \in \mathbb{R}^{n \times n}$, Lanczos basis $V_{n} \in \mathbb{R}^{N \times n}$.

1: Initialize $\bar{V}_{0} \leftarrow 0_{N, s}$ and $\bar{W}_{0} \leftarrow 0_{N, s}$ and compute

$$
\bar{V}_{1} \leftarrow\left[\boldsymbol{v}_{1}^{1}\left\|A \boldsymbol{v}_{1}^{1}\right\| \ldots \| A^{s-1} \boldsymbol{v}_{1}^{1}\right], \quad \bar{W}_{1} \leftarrow\left[\boldsymbol{w}_{1}^{1}\left\|A^{T} \boldsymbol{w}_{1}^{1}\right\| \ldots \|\left(A^{T}\right)^{s-1} \boldsymbol{w}_{1}^{1}\right] .
$$

2: Compute $2 s$ dot products $\left\langle\boldsymbol{w}_{1}^{1}, \boldsymbol{v}_{1}^{1}\right\rangle,\left\langle\boldsymbol{w}_{1}^{1}, A \boldsymbol{v}_{1}^{1}\right\rangle, \ldots,\left\langle\boldsymbol{w}_{1}^{1}, A^{2 s-1} \boldsymbol{v}_{1}^{1}\right\rangle$. 
3: Initialize $\left[\boldsymbol{b}_{0}^{1}\|\ldots\| \boldsymbol{b}_{0}^{s}\right] \leftarrow 0_{s, s},\left[\boldsymbol{c}_{0}^{1}\|\ldots\| \boldsymbol{c}_{0}^{s}\right] \leftarrow 0_{s, s},\left[\boldsymbol{d}_{0}^{1}\|\ldots\| \boldsymbol{d}_{0}^{s}\right] \leftarrow 0_{s, s}$, $\sigma_{1}^{1}, \ldots, \sigma_{1}^{s} \leftarrow 1,\left[\boldsymbol{t}_{0}^{2}\|\ldots\| \boldsymbol{t}_{0}^{s}\right] \leftarrow 0_{N, s}, \bar{M}_{0} \leftarrow 0_{s, s}$.

4: for $k=1$ until Convergence do

5: $\quad$ Compute for $i, j=1, \ldots, s$

$$
\begin{aligned}
\bar{M}_{k}^{i, j} & \leftarrow \sigma_{k}^{i} \sigma_{k}^{j}\left[\left\langle\boldsymbol{w}_{k}^{1}, A^{i+j-2} \boldsymbol{v}_{k}^{1}\right\rangle-\left(\boldsymbol{t}_{k-1}^{i}\right)^{T} \bar{M}_{k-1} \boldsymbol{t}_{k-1}^{j}\right], \\
\boldsymbol{c}_{k}^{s, j} & \leftarrow \sigma_{k}^{j}\left[\boldsymbol{b}_{k-1}^{s, j+1}-\left[\boldsymbol{d}_{k-1}^{s, 1}, \ldots, \boldsymbol{d}_{k-1}^{s, s}\right] \boldsymbol{t}_{k-1}^{j}\right] .
\end{aligned}
$$

6: $\quad$ Compute for $i, j=1, \ldots, s$

$$
\begin{aligned}
& \Omega_{k}^{i, j} \leftarrow\left(\boldsymbol{t}_{k-1}^{i}\right)^{T} \bar{M}_{k-1} \boldsymbol{t}_{k-1}^{j}, \quad \Omega_{k}^{s+1, j} \leftarrow \frac{\boldsymbol{c}_{k}^{s, s} \boldsymbol{t}_{k-1}^{s, j}}{\sigma_{k}^{s}}+\left(\boldsymbol{t}_{k-1}^{s}\right)^{T}\left[\boldsymbol{d}_{k-1}^{1}\|\ldots\| \boldsymbol{d}_{k-1}^{s}\right] \boldsymbol{t}_{k-1}^{j}, \\
& \Xi_{k}^{i, j} \leftarrow\left(\boldsymbol{t}_{k-1}^{i}\right)^{T} \bar{M}_{k-1} \boldsymbol{t}_{k-1}^{j}, \quad \Xi_{k}^{i, s+1} \leftarrow \frac{\boldsymbol{t}_{k-1}^{s, i} \boldsymbol{c}_{k}^{s, s}}{\sigma_{k}^{s}}+\left(\boldsymbol{t}_{k-1}^{i}\right)^{T}\left[\boldsymbol{d}_{k-1}^{1}\|\ldots\| \boldsymbol{d}_{k-1}^{s}\right] \boldsymbol{t}_{k-1}^{s} .
\end{aligned}
$$

7: $\quad$ Compute for $i, j=1, \ldots, s$

$$
\boldsymbol{d}_{k}^{i, j} \leftarrow \sigma_{k}^{i} \sigma_{k}^{j}\left[\left\langle\boldsymbol{w}_{k}^{1}, A^{i+j-1} \boldsymbol{v}_{k}^{1}\right\rangle-\Omega_{k}^{i+1, j}-\Xi_{k}^{i, j+1}+\left(\boldsymbol{t}_{k-1}^{i}\right)^{T}\left[\boldsymbol{d}_{k-1}^{1}\|\ldots\| \boldsymbol{d}_{k-1}^{s}\right] \boldsymbol{t}_{k-1}^{j}\right] .
$$

8: $\quad$ Solve $\bar{M}_{k-1} \boldsymbol{e}_{k}^{i}=\boldsymbol{c}_{k}^{i}$ and $\bar{M}_{k} \boldsymbol{g}_{k}^{i}=\boldsymbol{d}_{k}^{i}$ for all $i=1, \ldots, s$.

9: $\quad$ Compute $\tilde{\boldsymbol{v}}_{k+1}^{1} \leftarrow A \boldsymbol{v}_{k}^{s}-\bar{V}_{k-1} \boldsymbol{e}_{k}^{s}-\bar{V}_{k} \boldsymbol{g}_{k}^{s}$ and $\tilde{\boldsymbol{w}}_{k+1}^{1} \leftarrow A^{T} \boldsymbol{w}_{k}^{s}-\bar{W}_{k-1} \boldsymbol{e}_{k}^{s}-\bar{W}_{k} \boldsymbol{g}_{k}^{s}$.

10: $\quad$ Compute $A \tilde{\boldsymbol{v}}_{k+1}^{1}, A^{2} \tilde{\boldsymbol{v}}_{k+1}^{1}, \ldots, A^{s-1} \tilde{\boldsymbol{v}}_{k+1}^{1}$ and $A^{T} \tilde{\boldsymbol{w}}_{k+1}^{1},\left(A^{T}\right)^{2} \tilde{\boldsymbol{w}}_{k+1}^{1}, \ldots,\left(A^{T}\right)^{s} \tilde{\boldsymbol{w}}_{k+1}^{1}$.

11: $\quad$ Compute $2 s$ dot products $\left\langle\tilde{\boldsymbol{w}}_{k+1}^{1}, \tilde{\boldsymbol{v}}_{k+1}^{1}\right\rangle,\left\langle\tilde{\boldsymbol{w}}_{k+1}^{1}, A \tilde{\boldsymbol{v}}_{k+1}^{1}\right\rangle, \ldots,\left\langle\tilde{\boldsymbol{w}}_{k+1}^{1}, A^{2 s-1} \tilde{\boldsymbol{v}}_{k+1}^{1}\right\rangle$ and wait until global synchronization is completed.

12: $\quad$ Compute normalization coefficient $f_{k+1} \leftarrow \sqrt{\left|\left\langle\tilde{\boldsymbol{w}}_{k+1}^{1}, \tilde{\boldsymbol{v}}_{k+1}^{1}\right\rangle\right|}$.

13: $\quad$ Normalize $\boldsymbol{v}_{k+1}^{1} \leftarrow \tilde{\boldsymbol{v}}_{k+1}^{1} / f_{k+1}$ and $\boldsymbol{w}_{k+1}^{1} \leftarrow \tilde{\boldsymbol{w}}_{k+1}^{1} / f_{k+1}$.

14: Scale the already existing matrix-by-vector products $A \boldsymbol{v}_{k+1}^{1} \leftarrow A^{1} \tilde{\boldsymbol{v}}_{k+1}^{1} / f_{k+1}$, $\ldots, A^{s-1} \boldsymbol{v}_{k+1}^{1} \leftarrow A^{s-1} \tilde{\boldsymbol{v}}_{k+1}^{1} / f_{k+1}$ and $A^{T} \boldsymbol{w}_{k+1}^{1} \leftarrow A^{T} \tilde{\boldsymbol{w}}_{k+1}^{1} / f_{k+1},\left(A^{T}\right)^{s} \boldsymbol{w}_{k+1}^{1} \leftarrow\left(A^{T}\right)^{s} \boldsymbol{w}_{k+1}^{1} / f_{k+1}$.

15: Compute

$$
\left\langle\boldsymbol{w}_{k+1}^{1}, \boldsymbol{v}_{k+1}^{1}\right\rangle \leftarrow \frac{\left\langle\tilde{\boldsymbol{w}}_{k+1}^{1}, \tilde{\boldsymbol{v}}_{k+1}^{1}\right\rangle}{f_{k+1}^{2}}, \ldots,\left\langle\boldsymbol{w}_{k+1}^{1}, A^{2 s-1} \boldsymbol{v}_{k+1}^{1}\right\rangle \leftarrow \frac{\left\langle\tilde{\boldsymbol{w}}_{k+1}^{1}, A^{2 s-1} \tilde{\boldsymbol{v}}_{k+1}^{1}\right\rangle}{f_{k+1}^{2}},
$$

16: Compute normalization coefficients

$$
\sigma_{k+1}^{1} \leftarrow 1, \quad \sigma_{k+1}^{j} \leftarrow 1 / \sqrt{\left|\left\langle\boldsymbol{w}_{k+1}^{1}, A^{2 j-2} \boldsymbol{v}_{k+1}^{1}\right\rangle-\left(\boldsymbol{t}_{k}^{j}\right)^{T} \bar{M}_{k} \boldsymbol{t}_{k}^{j}\right|}, \text { for } j=2, \ldots, s .
$$

17: $\quad$ Compute for $i=1, \ldots, s$ and $j=2, \ldots, s+1$

$$
\boldsymbol{b}_{k}^{i, j} \leftarrow \frac{\sigma_{k}^{i}}{\sigma_{k}^{s}}\left[\left\langle f_{k+1} \boldsymbol{w}_{k+1}^{1}, A^{i+j-s-2} \boldsymbol{v}_{k+1}^{1}\right\rangle+\sum_{\substack{l=2 s \\+3-i-j}}^{s} \frac{\sigma_{k}^{\iota} \boldsymbol{g}_{k}^{\iota, s} \boldsymbol{b}_{k}^{i-s+\iota-1, j}}{\sigma_{k}^{i-s+\iota-1}}\right] .
$$

18: $\quad$ Solve $\bar{M}_{k} \boldsymbol{t}_{k}^{j}=\boldsymbol{b}_{k}^{j}$ for $j=2, \ldots, s$. 
19: $\quad$ Compute $\tilde{\boldsymbol{v}}_{k+1}^{j} \leftarrow A^{j-1} \boldsymbol{v}_{k+1}^{1}-\bar{V}_{k} \boldsymbol{t}_{k}^{j}$ for $j=2, \ldots, s$

and $\tilde{\boldsymbol{w}}_{k+1}^{j} \leftarrow\left(A^{T}\right)^{j-1} \boldsymbol{w}_{k+1}^{1}-\bar{W}_{k} \boldsymbol{t}_{k}^{j}$ for $j=2, \ldots, s$.

20: $\quad$ Normalize $\boldsymbol{v}_{k+1}^{j} \leftarrow \tilde{\boldsymbol{v}}_{k+1}^{j} \sigma_{k+1}^{j}$ and $\boldsymbol{w}_{k+1}^{j} \leftarrow \tilde{\boldsymbol{w}}_{k+1}^{j} \sigma_{k+1}^{j}$ for $j=2, \ldots, s$.

21: end for

22: Compute LU decomposition, $\ddot{L}_{n} \ddot{U}_{n}=\operatorname{diag}\left(\bar{M}_{1}, \ldots, \bar{M}_{m}\right)$.

23: Perform back transformation, $T_{n} \leftarrow \ddot{U}_{n} \ddot{T}_{n} \ddot{U}_{n}^{-1}, V_{n} \leftarrow \ddot{V}_{n} \ddot{U}_{n}^{-1}, W_{n}^{T} \leftarrow \ddot{L}_{n}^{-1} \ddot{W}_{n}^{T}$.

Table 1 compares the main computational cost for $n=s k$ iterations of the classical Lanczos algorithm and $k$ block iterations of two variants of the $s$-step Lanczos algorithm. More precisely, we report the number of operations as well as vector storages of size $N$ and neglect all corresponding costs of vectors of dimension $s$. Though the $s$-step variants slightly raise the computational cost, they reduce the number of global synchronization points by a factor of $\mathcal{O}(s)$.

Table 1. Comparison of cost for $n=s k$ iterations of the classical Lanczos algorithms and $k$ block iterations of the $s$-step Lanczos variants.

\begin{tabular}{llll}
\hline Operation/Storage & Classical & Unnormalized [16] & Normalized (Sect. 2) \\
\hline Dot products & $2 s k$ & $2 s k$ & $2 s k$ \\
Vector updates & $6 s k$ & $2 s(s-1) k+4 s k$ & $2 s(s-1) k+8 s k-k$ \\
Matrix-vector products & $2 s k$ & $2 s k+k$ & $2 s k+k$ \\
Synchronization points & $\mathcal{O}(s k)$ & $k$ & $k$ \\
Back transformations & - & $2 k$ & $2 k$ \\
Vector storage & 4 & $4 s$ & $4 s$ \\
\hline
\end{tabular}

\section{Numerical Experiment}

To compare the numerical behavior between the classical and the $s$-step Lanczos algorithm, we employ the following example taken from [16]. We consider the stationary two-dimensional convection-diffusion-reaction equation

$$
-\left(b u_{x}\right)_{x}-\left(c u_{x}\right)_{x}+(d u)_{x}+(e u)_{y}+f u=g \quad \text { on } \quad \Omega=(0,1) \times(0,1)
$$

with $b(x, y)=\mathrm{e}^{-x y}, c(x, y)=\mathrm{e}^{x y}, d(x, y)=x+y, e(x, y)=50(x+y), f(x, y)=$ $1 /(1+x+y)$ and Dirichlet boundary condition $u_{\mathrm{D}}=0$. This partial differential equation is discretized using first-order finite differences on an $n_{D} \times n_{D}$ grid leading to a system of linear equations whose coefficient matrix $A$ is of order $N=n_{D}^{2}=64^{2}=4096$ with the dominant eigenvalue $\lambda_{\max } \approx-17595+7170 \mathrm{i}$.

Figure 1 compares the Lanczos implementations. All algorithms are started with $\boldsymbol{v}_{1}^{1}=\boldsymbol{w}_{1}^{1}=[1, \ldots, 1]^{T}$. The upper diagram shows the convergence history of the relative accuracy of the dominant eigenvalue of $A$. The unnormalized 2-step variant starts to diverge at iteration 18 and breaks down at iteration 40, whereas the normalized 2-step variant competes well until iteration 61 . However, 
....... Classical — Unnormalized 2-step - - - Norm. 2-step - - - Norm. 5-step
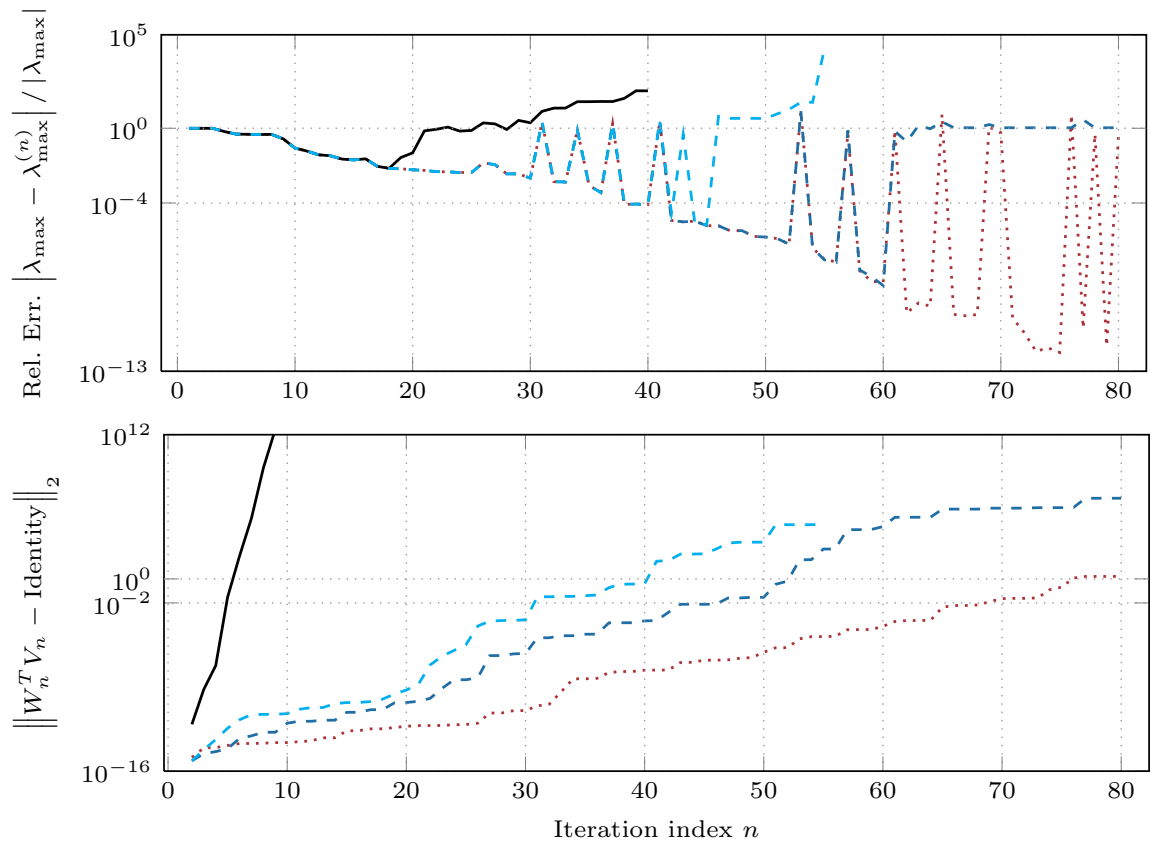

Fig. 1. Convergence history of the relative error of the dominant eigenvalue (top) and biorthogonality property (bottom) for the classical algorithm as well as for the unnormalized [16] and the normalized $s$-step Lanczos variant of Sect. 2.

when increasing $s$ to $s=5$, the divergence of the normalized variant already starts around iteration 45 . The tendency for growing numerical instabilities when increasing $s$ is well known $[18,19]$. The lower diagram gives the error of the biorthogonality property and shows a similar behavior of the algorithms.

\section{Parallel Performance}

Previous research $[16,20,21]$ already demonstrated that, compared to the classical algorithm, execution time is reduced by the unnormalized $s$-step algorithm. Therefore, we excluded the unnormalized $s$-step method from the analysis and only remark that the parallel performance characteristics of the normalized and unnormalized variants are almost identical. The focus is rather on the parallel performance of the normalized $s$-step Lanczos algorithm. To this end, we carried out a parallel implementation of that algorithm using PETSC [22] and included it as an additional eigenvalue solver inside SLEPC [23]. We compared the new normalized $s$-step variant to the classical variant implemented in SLEPC. 

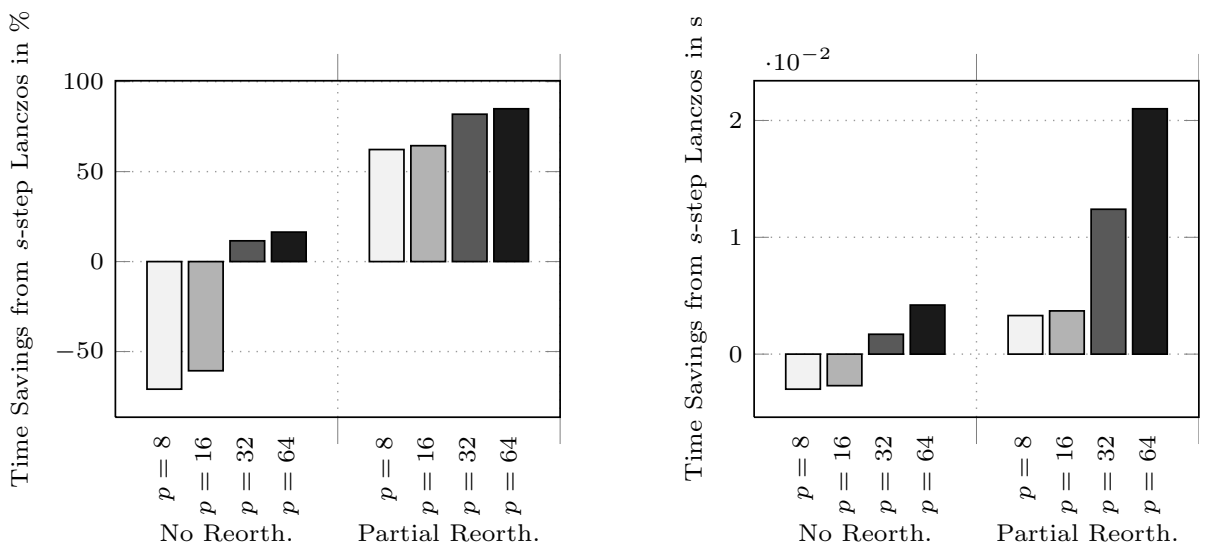

Fig. 2. Relative (left) and absolute (right) time savings per iteration, including time for reorthogonalization, when using the normalized $s$-step variant instead of the classical algorithm. We choose $s=2$ and the matrix from Sect. 3 using no and partial reorthogonalization and a different number of processes $p$.

When spurious eigenvalues are present, the Lanczos process can converge to wrong values. This behavior appears at the same time when the Lanczos vectors start to lose biorthogonality. As a possible remedy, one can explicitly reorthogonalize the new Lanczos vector with respect to previous ones. Our normalized $s$-step implementations can be used along with full, local, selective, periodic, and partial reorthogonalization schemes. However, reorthogonalization can only take place once after every block iteration of the $s$-step method. That is, one reorthogonalizes $\boldsymbol{v}_{k+1}^{1}$ and $\boldsymbol{w}_{k+1}^{1}$ against $V_{s k}$ and $W_{s k}$ (or columns thereof) in Step 13 of the algorithm. Each reorthogonalization comes at the cost of an additional GSP.

All computations are performed on a Nehalem-based Cluster at RWTH Aachen University, Germany. Each node of this cluster consists of 2 sockets, each equipped with Intel Xeon X5570 quadcore processors running at $2.93 \mathrm{GHz}$. Each core has a separate L1 and L2 cache; while 4 cores share an L3 cache of size $8 \mathrm{MB}$. So, each node of this cluster is made up of 8 cores called processes hereafter. The nodes are connected by a quad data rate InfiniBand network. A synchronization with a reduction operation accounts for $0.22 \times 10^{-4}$ s with 16 processes, $1.61 \times 10^{-4} \mathrm{~s}$ with 32 processes, and $3.38 \times 10^{-4} \mathrm{~s}$ with 64 processes.

Figure 2 compares the relative (left) and absolute (right) time savings per iteration when using the normalized $s$-step variant instead of the classical algorithm. In case of no reorthogonalization, the $s$-step algorithm performs slower than the classical algorithm for $p=8$ and $p=16$ processes. However, in this case it performs faster for both $p=32$ and $p=64$ processes. With 64 processes, for instance, the corresponding time saving per iteration accounts for $0.0042 \mathrm{~s}$ or $16.39 \%$ respectively. In case of partial reorthogonalization, the $s$-step variant saves time for all $p=8,16,32$, and 64 . Here, 64 processes result in time savings of $0.0210 \mathrm{~s}$ or $84.92 \%$ respectively. 


\section{Concluding Remarks}

In sparse linear algebra, the idea of $s$-step methods is to reduce the number of global synchronization points on distributed-memory computers by a factor of $\mathcal{O}(s)$. Rather than carrying out $s$ separate iterations of a traditional method, these methods rely on using a single block iteration that is equivalent in increasing the dimension of the Krylov subspace. We derive a new non-symmetric $s$-step Lanczos algorithm with normalization of the underlying Krylov basis. Numerical experiments indicate that this new variant - like the previous $s$-step variant-is more scalable than the traditional Lanczos algorithm. In addition, this new variant exhibits improved numerical accuracy compared to a previous $s$-step variant. So, this $s$-step Lanczos algorithm shows a possible path to advance parallel scalability on current large-scale and future extreme-scale supercomputers.

However, there is still room for further improvements. Most notably, the numerical stability tends to decrease with increasing $s$. Future work is necessary to investigate promising remedies such as residual replacement strategies or the use of a basis that is different from the monomial basis [5].

\section{Acknowledgements}

Parts of this research were conducted while the authors were in residence at the Institute for Scientific Computing, the Center for Computational Engineering Science, and the Aachen Institute for Advanced Study in Computational Engineering Science at RWTH Aachen University, D-52056 Aachen, Germany. Financial support from the Deutsche Forschungsgemeinschaft (German Research Foundation) through grant GSC 111 is gratefully acknowledged.

\section{References}

1. Lanczos, C.: An iteration method for the solution of the eigenvalue problem of linear differential and integral operators. J. Res. Nat. Bur. Stand. 45(4) (1950) $255-282$

2. Ghysels, P., Ashby, T.J., Meerbergen, K., Vanroose, W.: Hiding global communication latency in the GMRES algorithm on massively parallel machines. SIAM J. Sci. Comput. 35(1) (2013) C48-C71

3. Ghysels, P., Vanroose, W.: Hiding global synchronization latency in the preconditioned Conjugate Gradient algorithm. Parallel Computing (2013) In press.

4. Mohiyuddin, M., Hoemmen, M., Demmel, J., Yelick, K.: Minimizing communication in sparse matrix solvers. In: Proc. Conf. High Perf. Comput. Networking, Storage and Analysis. SC '09, New York, NY, USA, ACM (2009) 36:1-36:12

5. Carson, E., Knight, N., Demmel, J.: Avoiding communication in two-sided Krylov subspace methods. Technical Report UCB/EECS-2011-93, University of California, Berkeley (2011)

6. Fischer, B., Freund, R.: An inner product-free conjugate gradient-like algorithm for Hermitian positive definite systems. In Brown, J., et al., eds.: Proc. Cornelius Lanczos Intern. Centenary Conf., SIAM (1994) 288-290 
7. Meurant, G.: The conjugate gradient method on supercomputers. Supercomputer 13 (1986) 9-17

8. Van Rosendale, J.: Minimizing inner product data dependencies in conjugate gradient iteration. NASA Contractor Report NASA-CR-172178, NASA Langley Research Center, Hampton, VA (1983)

9. Bücker, H.M., Sauren, M.: A Variant of the Biconjugate Gradient Method Suitable for Massively Parallel Computing. In Bilardi, G., et al., eds.: Solving Irregularly Structured Problems in Parallel, Proc. IRREGULAR'97, Paderborn, Germany, June 12-13, 1997. Volume 1253 of LNCS., Berlin, Springer (1997) 72-79

10. Bücker, H.M., Sauren, M.: A Parallel Version of the Quasi-Minimal Residual Method Based on Coupled Two-Term Recurrences. In Waśniewski, J., et al., eds.: Applied Parallel Computing, Proc. PARA '96, Lyngby, Denmark, August 18-21, 1996. Volume 1184 of LNCS., Berlin, Springer (1996) 157-165

11. Bücker, H.M., Sauren, M.: Reducing global synchronization in the biconjugate gradient method. In Yang, T., ed.: Parallel numerical computations with applications. Kluwer Academic Publishers, Norwell, MA, USA (1999) 63-76

12. Chronopoulos, A.T.: A Class of Parallel Iterative Methods Implemented on Multiprocessors. Technical report UIUCDCS-R-86-1267, Department of Computer Science, University of Illinois, Urbana, Illinois (1986)

13. Chronopoulos, A.T., Gear, C.W.: s-step iterative methods for symmetric linear systems. J. Comput. Appl. Math. 25(2) (1989) 153-168

14. Chronopoulos, A.T., Swanson, C.D.: Parallel iterative s-step methods for unsymmetric linear systems. Parallel Computing 22(5) (1996) 623-641

15. Kim, S.K., Chronopoulos, A.: A class of Lanczos-like algorithms implemented on parallel computers. Parallel Computing 17(6-7) (1991) 763-778

16. Kim, S.K., Chronopoulos, A.T.: An efficient nonsymmetric Lanczos method on parallel vector computers. J. Comput. Appl. Math. 42(3) (1992) 357-374

17. Feuerriegel, S.: Lanczos-based Algorithms for the Parallel Solution of Large Sparse Linear Systems. Master's thesis, RWTH Aachen University, Aachen (2011)

18. Kim, S.K.: Efficient biorthogonal Lanczos algorithm on message passing parallel computer. In Hsu, C.H., Malyshkin, V., eds.: Proc. MTPP 2010. Volume 6083 of LNCS., Berlin, Springer (2010) 293-299

19. Carson, E., Demmel, J.: A residual replacement strategy for improving the maximum attainable accuracy of s-step Krylov subspace methods. Technical Report UCB/EECS-2012-197, University of California, Berkeley (2012)

20. Gustafsson, M., Kormann, K., Holmgren, S.: Communication-efficient algorithms for numerical quantum dynamics. In Jónasson, K., ed.: Applied Parallel and Scientific Computing, Proc. PARA 2010, Reykjavik, Iceland, June 6-9, 2010. Volume 7134 of LNCS., Berlin, Springer (2012) 368-378

21. Kim, S.K., Kim, T.H.: A study on the efficient parallel block Lanczos method. Computational and Information Science (2005) 231-237

22. Balay, S., Gropp, W.D., McInnes, L.C., Smith, B.F.: Efficient management of parallelism in object oriented numerical software libraries. In Arge, E., et al., eds.: Modern Software Tools in Scientific Computing, Birkhäuser Press (1997) 163-202

23. Hernandez, V., Roman, J.E., Vidal, V.: SLEPc: A scalable and flexible toolkit for the solution of eigenvalue problems. ACM Trans. Math. Softw. 31(3) (2005) $351-362$ 